\title{
Optimal Tracking Using Magnetostrictive Actuators Operating in Nonlinear and Hysteretic Regimes
}

\author{
William S. Oates ${ }^{1}$ and Ralph C. Smith ${ }^{2}$ \\ Center for Research in Scientific Computation \\ Department of Mathematics \\ North Carolina State University \\ Raleigh, NC 27695
}

\begin{abstract}
Many active materials exhibit nonlinearities and hysteresis when driven at field levels necessary to meet stringent performance criteria in high performance applications. This often requires nonlinear control designs to effectively compensate for the nonlinear, hysteretic field-coupled material behavior. In this paper, an optimal control design is developed to accurately track a reference signal using magnetostrictive transducers. The methodology can be directly extended to transducers employing piezoelectric materials or shape memory alloys (SMAs) due to the unified nature of the constitutive model employed in the control design. The constitutive model is based on a framework that combines energy analysis at lattice length scales with stochastic homogenizations techniques to predict macroscopic material behavior. The constitutive model is incorporated into a finite element representation of the magnetostrictive transducer which provides the framework for developing the finite-dimensional nonlinear control design. The control design includes an open loop nonlinear component computed off-line with perturbation feedback around the optimal state trajectory. Estimation of unmeasurable states is achieved using a Kalman filter. The hybrid control technique provides the potential for real-time control implementation while providing robustness with regard to operating uncertainties and unmodeled dynamics.
\end{abstract}

Keywords: nonlinear optimal tracking, magnetostrictive actuators, perturbation control, Kalman filter

\section{Introduction}

Smart materials and structures play an important role in improving performance capabilities of aeronautic, aerospace, automotive, industrial, biomedical, and military systems. The need for high power density devices that can operate over a broad frequency range with precise control continues to draw interests in transducer

\footnotetext{
${ }^{1}$ Email: wsoates@ncsu.edu, Telephone: (919) 515-2386

2Email: rsmith@eos.ncsu.edu, Telephone: (919) 515-7552
} 
designs that employ smart materials such as piezoelectric materials, magnetostrictive compounds and shape memory alloys (SMAs). For example, piezoelectric materials offer actuating properties with nanoscale resolution and large actuation forces over a broad range of frequencies (Hz-MHz). This provides advantages in numerous applications such as high resolution acoustic imaging [1], morphing aircraft control surfaces [2], and high resolution nanopositioning stages $[3,4]$. High force magnetostrictive materials such as Terfenol-D provide broadband capability (DC up to $20 \mathrm{kHz}$ ) with forces up to $550 \mathrm{~N}[5,6]$, which are utilized in many industrial settings such as high-speed precision machining [7]. A good review of numerous magnetostrictive device applications is given in [8]. Shape memory alloys operate at lower frequencies $(<100 \mathrm{~Hz})$ but with relatively large power density which provides certain advantages in applications such as morphing aircraft structures for improved aerodynamic performance $[9,10]$ and reduction of jet engine noise [11].

Whereas smart material devices offer several advantages over conventional actuation systems, challenges associated with achieving accurately controlled dynamics require careful characterization and control of the field-coupled material behavior. In the context of piezoelectric and magnetostrictive materials, a small input field results in a manifestation of local polarization or magnetic variants moving in an approximately reversible manner creating macroscopic strains, which are approximately linear. Although linear models and control theory often provide reasonable approximations when the input fields are small, moderate to large fields are often necessary to meet stringent performance criteria. These field levels typically change the internal state of the material in a nonlinear and irreversible manner which complicates models and control designs. If this behavior is neglected, degradation in control authority may result from unmodeled constitutive nonlinearities and potential instabilities from a phase lag between the input field and actuator displacement. It is often necessary to introduce the nonlinear and hysteretic material behavior into the control design via either inverse filters used to linearize the transducer response $[7,12]$ or direct nonlinear compensation using nonlinear control designs $[13,14,15,16]$. Whereas the use of inverse filters can improve tracking performance by effectively compensating for nonlinear and irreversible material behavior, the interpretation of the transducer input is more difficult due to the inversion of the constitutive law. This complexity can often be avoided by the use of nonlinear control design which circumvents the need for an inverse filter and directly determines the nonlinear control input. The inverse filter and nonlinear control design approaches are illustrated in Fig. 1.

Model-based control designs for hysteretic systems are not new and have received considerable attention in areas related to adaptive, classical, optimal and robust control [7, 12, 13, 14, 17, 18, 19]. These models often address hysteretic material behavior by implementing Preisach operators [12] or domain wall models [20]. Although Preisach models can reasonably predict nonlinear and hysteretic material behavior observed in smart materials such as piezoelectric, magnetostrictive and shape memory alloys, a significant number of 


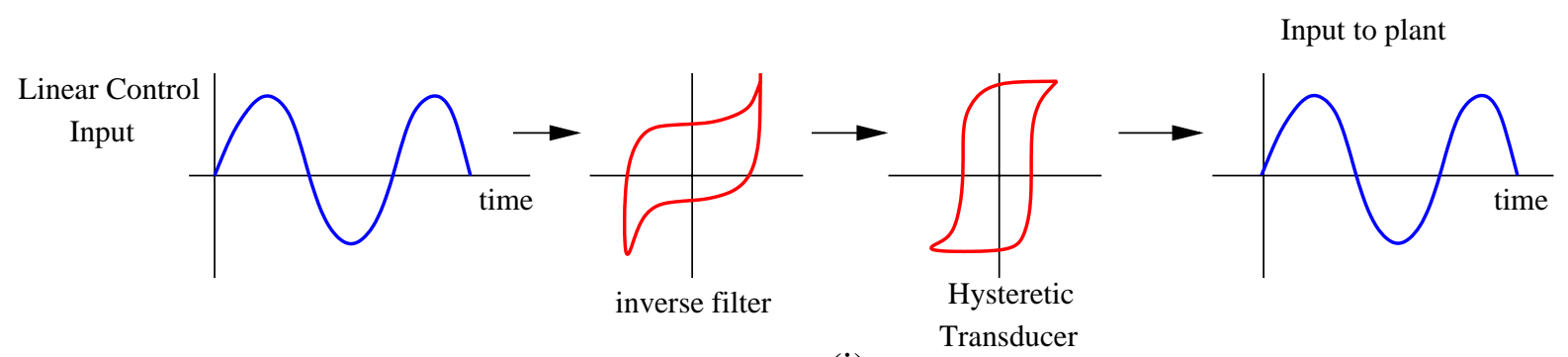

(i)

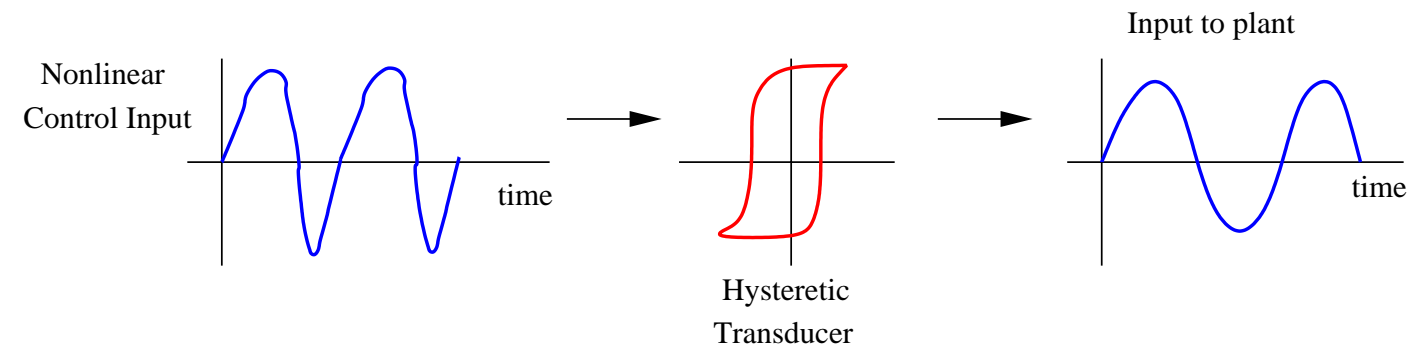

(ii)

Figure 1: (i) Linear control design employing an inverse filter, and (ii) nonlinear control design.

non-physical parameters are necessary to model minor loop hysteresis. Moreover, significant extensions to the classical Preisach theory are required to accommodate relaxation, reversible behavior, and frequency-dependent dynamics. Domain wall models avoid some of these issues by incorporating energetics at the domain length scale to reasonably predict unbiased macroscopic hysteretic material behavior. However, magnetostrictive transducers are typically magnetized by a permanent magnet to provide improved actuation properties [5]. Similarly, piezoelectric actuators are often biased with a static electric field to allow increases in the applied unipolar field and corresponding strain without inducing reverse ferroelectric switching. In these cases, rate independent biased minor loops typically exhibit closure properties which cannot be effectively predicted without extensive modifications of the domain wall model. In the magnetization model developed in [21], closure of biased minor loops was enforced by a priori knowledge of the turning points which precludes use in feedback control since the states are not known in advance. When inaccuracies due to nonclosure are significant, Preisach models or homogenized energy models are typically employed in the control design.

The present analysis employs the homogenized energy framework [22, 23, 24, 25] in the control design to characterize various mechanisms contributing to nonlinear and hysteretic magnetostrictive material behavior. The control design is demonstrated for a magnetostrictive transducer used to accurately machine an out-of-round automotive part at high speed. This application requires a $100 \mu \mathrm{m}$ displacement peak-to-peak with accuracy of $1 \mu \mathrm{m}-2 \mu \mathrm{m}$ at speeds of approximately $3000 \mathrm{rpm}$. This approach is similar to a previous investigation of high 
speed and high accuracy machining applications [7] that utilized an inverse filter to linearize the transducer response. The inverse filter was implemented in a robust control design using both $H_{2}$ and $H_{\infty}$ control designs to accurately track the desired profile. The control design developed here directly determines the nonlinear control input by implementing the magnetostrictive constitutive behavior into the control design. Furthermore, the control input is determined using optimal control techniques that extend recent developments in hybrid nonlinear optimal vibration control of plate structures [14]. The previous work utilized a nonlinear open loop control component together with linear perturbation feedback to improve robustness to operating uncertainties in actively attenuating plate vibration with magnetostrictive transducers. The control design presented here required modification of the perturbation feedback control when tracking a reference signal. Additionally, certain states in the system under consideration are assumed to be unmeasurable which motivated implementing a Kalman filter to estimate unobservable states in the perturbed optimality system. This approach provides a potential method for real-time state feedback of perturbations around the open loop nonlinear control for high performance tracking applications.

The system model and control design are developed as follows. First, the constitutive model is briefly summarized. A structural model is then developed using one-dimensional finite elements to couple the magnetostrictive transducer to a damped oscillator used to represent boundary conditions between the transducer and the part being machined. The control theory is then developed. Tracking performance is demonstrated using linear optimal control theory and compared to the nonlinear optimal control approach. Significant improvements in accurate position control are obtained when the magnetostrictive constitutive behavior is compensated using the open loop nonlinear control. To improve robustness to various operating uncertainties, perturbation feedback is implemented in the final section. A linear Kalman filter is introduced into the perturbed optimality system to estimate unobservable states in the transducer. It is demonstrated that significant improvements in control authority are achieved when using the perturbation feedback in concert with the Kalman filter.

\section{Magnetostrictive Transducer and Hysteresis Model}

Magnetostrictive materials deform when exposed to magnetic fields due to coupling between magnetic moment orientations and elastic interactions. A fundamental challenge in effectively utilizing these materials in actuator applications deals with accurate prediction of field-coupled constitutive nonlinearities and irreversibilities. The primary mechanism responsible for nonlinearities and hysteresis is domain wall motion and rotation around pinning sites from various inhomogeneities such as impurities, inclusions and local residual stress [26]. Whereas this behavior can be mitigated by applying only small fields, moderate to high input fields are typically necessary in high performance applications. This requires accommodating constitutive nonlinearities and hysteresis in the control design so that large strain and high force capabilities can be effectively utilized. 


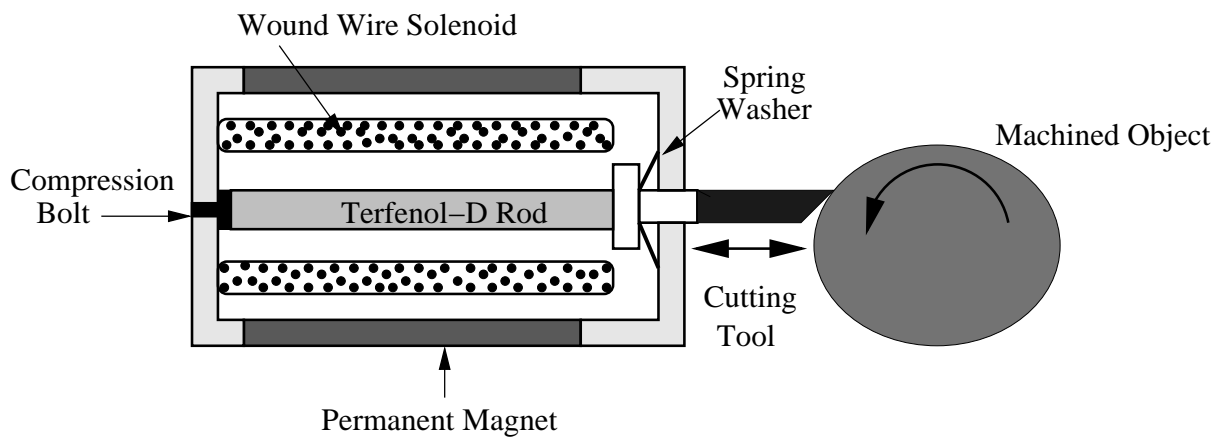

Figure 2: Terfenol-D transducer design used for high accuracy machining operations of cutting out-of-round objects.

A commonly used magnetostrictive material for actuator applications is Terfenol-D $\left(\mathrm{Tb}_{0.3} \mathrm{Dy}_{0.7} \mathrm{Fe}_{1.9-1.95}\right)$. This composition is typically employed in transducer designs due to its broadband capability (DC up to $20 \mathrm{kHz}$ ), large strain $(1,600 \mu \epsilon)$ and large force output $(550 \mathrm{~N})[27,5]$. This material is often manufactured as monolithic rods for incorporating the material into a typical transducer design as depicted in Fig. 2. The transducer primarily consists of the Terfenol-D rod, a surrounding wound wire solenoid, a compression bolt/spring washer assembly, and a permanent magnet. A current is supplied to the wound wire solenoid which produces a magnetic field in the Terfenol-D rod. Magnetic flux, magnetization and strains are induced by the magnetic field. The compression bolt/spring washer assembly prestresses the Terfenol-D rod to avoid tensile loads during operation and increased actuator displacement [28]. The permanent magnet serves to bias the magnetization in the Terfonol-D rod which produces uniform flux patterns in the rod and bi-directional strains from a time-varying magnetic field input with zero DC offset. This avoids the ohmic heating associated with DC bias fields, but increases the size and weight of the transducer.

\subsection{Homogenized Energy Model}

The homogenized energy model is based on a multi-scale modeling approach that assumes an underlying distribution of material property relations at the mesoscopic length scale rather than assuming uniform material properties represented by material constants. A stochastic homogenization technique is implemented to determine macroscopic thermodynamic parameters from the distribution of the local material properties. The governing equations pertaining to the control design are summarized here. A detailed review of the model for ferromagnetic materials is given in $[22,24]$.

We focus on uniaxial loading of rod-type actuators and thus assume that fields and material coefficients have been reduced to scalar coefficients or distributed variables in the direction of loading. The Gibbs energy at the 
mesoscopic length scale is

$$
G(M, T)=\Psi(M, T)-\mu_{0} H M
$$

where $\Psi(M, T)$ is the Helmholtz energy detailed in [22], $\mu_{0}$ is the permeability of free space, $T$ is temperature, $H$ is the magnetic field, and $M$ is the magnetization. In the one-dimensional case considered here, the Helmholtz energy yields a double-well potential below the Curie point $T_{c}$ which gives rise to stable spontaneous magnetization with equal magnitude in the positive or negative directions.

In certain cases, thermal activation mechanisms are significant and must be included in the constitutive model. This can be accomplished by introducing the Boltzman relation

$$
\mu(G)=C e^{-G V / k T}
$$

which quantifies the propability $\mu$ of achieving an energy level $G$. Here $k T / V$ is the relative thermal energy where $V$ is a representative volume element at the mesoscopic length scale, $k$ is Bolztmann's constant, and the constant $C$ is specified to ensure integration to unity. From a physical perspective, large thermal energy relative to the Gibbs energy reduces the barrier for magnetization variants to switch when the applied field is near the coercive field. This reduces the sharp transition of magnetic variants switching from the positive to negative state and vice versa.

The Boltzman relation is utilized to determine the expected magnetization variants governed by

$$
\left\langle M_{+}\right\rangle=\int_{M_{I}}^{\infty} M \mu(G) d M \quad, \quad\left\langle M_{-}\right\rangle=\int_{-\infty}^{-M_{I}} M \mu(G) d M
$$

where $\pm M_{I}$ are the positive and negative inflection points in the Helmholtz energy definition.

The local magnetization is subsequently defined by

$$
\bar{M}=x_{+}\left\langle M_{+}\right\rangle+x_{-}\left\langle M_{-}\right\rangle
$$

where $x_{+}$and $x_{-}$respectively denote the volume fraction of magnetization variants having positive and negative orientations. The differential equations governing the evolution of $x_{+}$and $x_{-}$are based on material-dependent parameters that define the likelihoods that moments switch from positive to negative, and conversely. These likelihoods account for the observed thermal relaxation mechanisms. Details describing the governing equations are given in $[22,24]$. 
It has been shown [22] that in cases when relative thermal effects are negligible ( $k T / V$ is small) and relaxation times are small compared to the drive frequencies, the relation Eq. (4) limits to the piecewise linear relation

$$
\bar{M}\left(H+H_{I} ; H_{c}, \xi\right)=\chi_{m}\left(H+H_{I}\right)+\delta M_{R}
$$

where $\chi_{m}$ is the magnetic susceptibility, $M_{R}$ denotes the local magnetization variant and $\delta=1$ for local variants aligned in the positive direction and $\delta=-1$ for local variants aligned in the negative direction. The interaction field $H_{I}$ represents local variations in the field due to material inhomogeneities. The local remanence magnetization $M_{R}$ will switch when the diametrically opposed effective field $\left(H_{e}=H+H_{I}\right)$ reaches the coercive field. A semi-infinite set of magnetic variants, interaction fields and coercive fields $H_{c}$ are used to determine when each variant switches. Details regarding the numerical procedure are given in [22].

The macroscopic magnetization is computed from the distribution of local variants via the relation

$$
[M(H)](t)=\int_{0}^{\infty} \int_{-\infty}^{\infty} \nu\left(H_{c}, H_{I}\right)\left[\bar{M}\left(H+H_{I} ; H_{c}, \xi\right)\right](t) d H_{I} d H_{c}
$$

where $\xi$ represents the initial distribution of the local variants, and $\nu\left(H_{c}, H_{I}\right)$ denotes the distribution of coercive and interaction fields. To facilitate parameter estimation, the following form of the distribution is used

$$
\nu\left(H_{c}, H_{I}\right)=c_{1} e^{-\left[\ln \left(H_{c} / \bar{H}_{c}\right) / 2 c\right]^{2}} e^{-H_{I}^{2} / 2 b^{2}}
$$

where $\bar{H}_{c}$ is the average coercive field, $c$ quantifies the coercive field variability, $b$ is the variance of the interaction field, and $c_{1}$ is a scaling parameter. Model comparison to experimental results can be found in [22].

For implementing the magnetostrictive material behavior within the control design, the forces generated by the transducer must be quantified. This is provided by the constitutive law

$$
\sigma=Y^{M} \varepsilon-a_{1}\left(M(H)-M_{0}\right)^{2}
$$

representing uniaxial stress in the Terfenol-D rod where $Y^{M}$ is the elastic modulus at constant magnetization, $\varepsilon$ is the linear strain component, and $a_{1}$ is the magnetostrictive coefficient. It is assumed that stress fields are limited to the linear elastic regime where ferroelastic switching is negligible. Here, $M(H)$ is the magnetization computed in Eq. (6) whereas $M_{0}$ includes effects from the permanent magnet and the initial magnetized state of the material.

The stress computed using Eq. (8) includes linear stress-strain behavior as well as nonlinear and hysteretic dependence on the magnetic field through the $M(H)$ relation. It does not include internal damping or spatial dependence. This is incorporated in the following section. 


\section{Structural Model}

To facilitate the control design, the constitutive relations given by Eqs. (6) and (8) are used to develop a system model that quantifies forces and displacements when a magnetic field or stress is applied to the magnetostrictive transducer. The PDE model is first presented and then formulated as an ODE system through a finite element discretization in space. A damped oscillator serves as boundary conditions at the end of the actuator to represent the structure being machined. The structural model is illustrated in Fig. 3.

A balance of forces for the structural model is given by the relation [29]

$$
\rho A \frac{\partial^{2} w}{\partial t^{2}}=\frac{\partial N_{t o t}}{\partial x}
$$

where the density of the actuator is denoted by $\rho$, the cross-section area is $A$ and the displacement is denoted by $w$. The total force $N_{t o t}$ acting on the actuator is given by the relation

$$
N_{t o t}(t, x)=Y^{M} A \frac{\partial w}{\partial x}+c_{D} A \frac{\partial^{2} w}{\partial x \partial t}+F_{m a g}(H)+F_{d}
$$

where the elastic restoring force is given by the first term on the right hand side of the equation and a KelvinVoigt damping force is given by the second term. The linear elastic strain component is defined by $\varepsilon=\frac{\partial w}{\partial x}$. In comparison to Eq. (8), the total force acting on the Terfenol-D rod includes the additional internal damping force and the external disturbance load $F_{d}$. The coupling force $F_{m a g}$ represents forces generated by an applied magnetic field where

$$
F_{\text {mag }}(H)=A a_{1}\left(M(H)-M_{0}\right)^{2}
$$

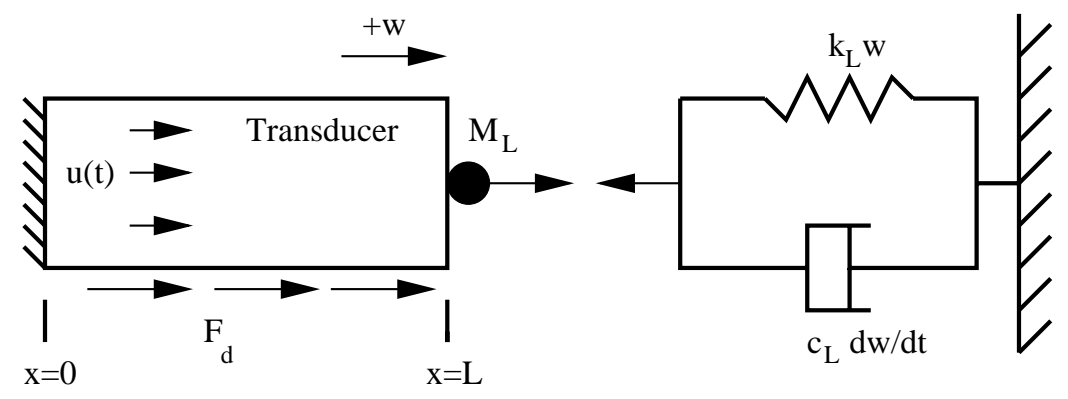

Figure 3: Magnetostrictive transducer with damped oscillator used to quantify loads during the maching operation. Disturbance forces along the actuator are given by $F_{d}$ and the control input is $u(t)$. 
As illustrated in Fig. 3, the boundary conditions are defined by a zero displacement at $x=0$ and the balance of forces at $x=L$ given by

$$
N_{t o t}(t, L)=-k_{L} w(t, L)-c_{L} \frac{\partial w}{\partial t}(t, L)-m_{L} \frac{\partial^{2} w}{\partial t^{2}}(t, L)
$$

The initial conditions are $w(0, x)=0$ and $\frac{\partial w}{\partial x}(0, x)=0$.

The strong form of the PDE model given by Eq. (9) can be written in the variational or weak form for finite element implementation. Multiplication by weight functions followed by integration yields

$$
\begin{aligned}
\int_{0}^{L} \rho A \frac{\partial^{2} w}{\partial t^{2}} \phi d x= & -\int_{0}^{L}\left[Y^{M} A \frac{\partial w}{\partial x}+c_{D} A \frac{\partial^{2} w}{\partial x \partial t}+F_{m a g}(H)+F_{d}\right] \frac{\partial \phi}{\partial x} d x \\
& -\left[k_{L} w(t, L)+c_{L} \frac{\partial w}{\partial t}(t, L)+m_{L} \frac{\partial^{2} w}{\partial t^{2}}(t, L)\right] \phi(L)
\end{aligned}
$$

where the weight functions $\phi(x)$ are required to satisfy the essential boundary condition $w(t, 0)=0$ but not the natural boundary condition given by Eq. (12) at $x=L$. The weak form of the model given by Eq. (13) is used to obtain a matrix ODE system.

\subsection{Approximation Method}

An approximate solution to Eq. (13) is obtained by discretizing the rod in space followed by a finite-difference approximation of the resulting temporal system. The transducer is partitioned into equal segments over the length $[0, L]$ with points $x_{i}=i h, i=0,1, \ldots, N$ and step size $h=L / N$, where $N$ denotes the number of finite elements. The spatial basis $\left\{\phi_{i}\right\}_{i=1}^{N}$ is comprised of the linear interpolation functions

$$
\phi_{i}(x)=\frac{1}{h} \begin{cases}\left(x-x_{i-1}\right), & x_{i-1} \leq x<x_{i} \\ \left(x_{i+1}-x\right), & x_{i} \leq x<x_{i+1} \\ 0, & \text { otherwise }\end{cases}
$$

for $i=1, \ldots, N-1$. For $i=N$, the basis function is defined to be

$$
\phi_{N}(x)=\frac{1}{h} \begin{cases}\left(x-x_{N-1}\right), & x_{N-1} \leq x<x_{N} \\ 0, & \text { otherwise. }\end{cases}
$$

Details describing the interpolation functions are given in [22].

The approximate solution to Eq. (13) is given by a linear superposition of the interpolation functions 


$$
w^{N}(t, x)=\sum_{j=1}^{N} w_{j}(t) \phi_{j}(x)
$$

where $w_{j}(t)$ are the nodal displacement solutions along the length of the transducer.

An ODE matrix system is obtained by substituting $w^{N}(t, x)$ into Eq. (13) with the interpolation functions employed as the weight functions. This gives rise to a second-order matrix equation

$$
\mathbb{M} \ddot{\vec{w}}+\mathbb{C} \dot{\vec{w}}+\mathbb{K} \vec{w}=F_{m a g}(H) \vec{b}+\vec{f}_{d}
$$

where the displacement solution $w_{j}(t)$ has been written in vector form $\vec{w}(t)=\left[w_{1}(t), \ldots, w_{N}(t)\right]$ and $\mathbb{M} \in \mathbb{R}^{N \times N}$, $\mathbb{C} \in \mathbb{R}^{N \times N}$ and $\mathbb{K} \in \mathbb{R}^{N \times N}$ denote the mass, damping and stiffness matrices. The vectors $\vec{b} \in \mathbb{R}^{N}$ and $\vec{f}_{d} \in \mathbb{R}^{N}$ include the integrated interpolation functions related to the control input and disturbance loads, respectively. Components contained within the system matrices and vectors are given in the Appendix.

Formulation of Eq. (17) as a first order system yields

$$
\begin{aligned}
& \dot{x}(t)=A x(t)+[B(u)](t)+G(t) \\
& x(0)=x_{0}
\end{aligned}
$$

where $x(t)=[\vec{w}, \dot{\vec{w}}]^{T}$ should not to be confused with the coordinate $x$. The matrix $A$ incorporates the mass, damping and stiffness matrices given in Eq. $(17)$ and $[B(u)](t)$ includes the nonlinear input where $u(t)$ is defined as the magnetic field to be consistent with the control literature. External disturbances are given by $G$. The system matrix $A$, input vector $B(u)$ and disturbance load $G(t)$ are

$$
A=\left[\begin{array}{cc}
0 & \mathbb{I} \\
-\mathbb{M}^{-1} \mathbb{K} & -\mathbb{M}^{-1} \mathbb{C}
\end{array}\right] \quad, \quad B(u)=F_{m a g}(u)\left[\begin{array}{c}
0 \\
\mathbb{M}^{-1} \vec{b}
\end{array}\right] \quad, \quad G(t)=\left[\begin{array}{c}
0 \\
\mathbb{M}^{-1} \vec{f}_{d}(t)
\end{array}\right]
$$

The identity matrix, with dimension $N \times N$, is denoted by $\mathbb{I}$.

The system given by Eq. (18) must be discretized in time for simulation purposes. Here, the trapezoid rule is adopted since it is moderately accurate, A-stable, and requires minimal computer storage capacity. A standard trapezoidal discretization yields the iteration

$$
\begin{aligned}
& x_{k+1}=\mathbb{W} x_{k}+\frac{1}{2} \mathbb{V}\left[B\left(u_{k}\right)+B\left(u_{k+1}\right)+G\left(t_{k}\right)+G\left(t_{k+1}\right)\right] \\
& x(0)=x_{0}
\end{aligned}
$$


where a temporal step size $\Delta t$ is employed giving a discretization in time defined by $t_{k}=k \Delta t$. The values $x_{k}$ approximate $x\left(t_{k}\right)$. The matrices

$$
\begin{aligned}
& \mathbb{W}=\left[\mathbb{I}-\frac{\Delta t}{2} A\right]^{-1}\left[\mathbb{I}+\frac{\Delta t}{2} A\right] \\
& \mathbb{V}=\Delta t\left[\mathbb{I}-\frac{\Delta t}{2} A\right]^{-1}
\end{aligned}
$$

are created once for numerical implementation yielding approximate solutions with $O\left(h^{2},(\Delta t)^{2}\right)$ accuracy.

\section{Control Design}

We first outline the general tracking problem to provide relations used when developing the nonlinear optimal tracking law. The tracking problem requires defining the output of the transducer system. This is developed by assuming that the position of the cutting tool depicted in Fig. (2) can be measured and all other states are unobservable. The output to Eq. (18) is represented by

$$
y(t)=C x(t), \quad x \in \mathbb{R}^{2 N}
$$

where $C=[0, \ldots, 0,1,0, \ldots, 0]$ has dimension $1 \times 2 N$. The cost functional

$$
\bar{J}=\frac{1}{2}\left(C x\left(t_{f}\right)-r\left(t_{f}\right)\right)^{T} P\left(C x\left(t_{f}\right)-r\left(t_{f}\right)\right)+\int_{t_{0}}^{t_{f}}\left[H-\lambda^{T}(t) \dot{x}(t)\right] d t
$$

is defined to penalize the control input and the error between the measurable state and the reference signal $r(t)$ where $P$ penalizes large terminal values on the observable state, $H$ is the Hamiltonian, and $\lambda(t)$ is a set of Lagrange multipliers. The Hamiltonian is,

$$
\begin{aligned}
H= & \frac{1}{2}\left[(C x(t)-r(t))^{T} Q(C x(t)-r(t))+u^{T}(t) R u(t)\right] \\
& +\lambda^{T}[A x(t)+[B(u)](t)+G(t)]
\end{aligned}
$$

where penalities on the observable state and the input are given through the variables $Q$ and $R$, respectively.

Through the construction of $C$, unobservable states are not included in the performance index. The cost functional can be augmented to include the additional states without conflict in performance objectives [30], but this is not considered in the open loop control design. This issue is treated using perturbation feedback with state estimation in Section 4.3 to minimize potentially undesirable variations in unobservable states. 
To determine the optimal input, the minumum of Eq. (23) must be determined under the constraint of Eq. (18) which is incorporated via the Lagrange multipliers. As detailed in [31, 32], the stationary condition for the Hamiltonian yields the adjoint relation

$$
\dot{\lambda}(t)=-A^{T} \lambda(t)-C^{T} Q C x(t)+C^{T} Q r(t)
$$

and control condition

$$
u(t)=-R^{-1}\left(\frac{\partial B(u)}{\partial u}\right)^{T} \lambda(t)
$$

The boundary conditions are applied to the state at the initial time and the costate at the final time

$$
\begin{aligned}
& x\left(t_{0}\right)=x_{o} \\
& \lambda\left(t_{f}\right)=C^{T} P\left(C x\left(t_{f}\right)-r\left(t_{f}\right)\right) .
\end{aligned}
$$

These equations result in a two-point boundary value problem that is, in general, challenging to solve for large systems. In the following section, linear control theory is implemented to illustrate that poor control authority occurs when the field input approaches the moderate to large regime. The degradation in control authority is then addressed using the nonlinear control design. It is demonstrated that by directly employing the constitutive model in the optimality system and solving the two-point boundary value problem through a combination of analytical and numerical techniques, significant improvements in tracking a reference signal are obtained. In the last section, improvements in robustness to operating uncertainties are realized by implementing perturbation feedback around the optimal open loop trajectory with feedback of estimated states via a linear Kalman filter.

\subsection{Linear Tracking Control}

The linear control design requires simplifying the nonlinear input given by the coupling force in Eq. (11). The force is linearized by taking a Taylor series expansion about the fully magnetized state of the material. The control input in this case is limited to a small time varying input, which requires only a consideration of first order variations in the magnetic force.

Linearization about $M_{0}$ yields the force relation

$$
F_{m a g} \simeq 2 A a_{1} M_{0} \Delta M
$$

where $\Delta M=M-M_{0}$. 
A linear magnetic constitutive law is introduced so that the control input is given by the applied magnetic field

$$
\Delta M=M-M_{0}=\chi_{m} H
$$

where $H$ is the change in magnetic field from a zero DC offset. This yields

$$
F_{m a g} \simeq 2 A a_{1} M_{0} \chi_{m} H
$$

With this approximation, the corresponding first-order system is

$$
\begin{aligned}
& \dot{x}(t)=A x(t)+B u(t)+G(t) \\
& x(0)=x_{0}
\end{aligned}
$$

where $u(t)$ denotes the magnetic field.

The state constraint in Eq. (31) and the adjoint condition in Eq. (25) yields the optimality system

$$
\begin{aligned}
& {\left[\begin{array}{c}
\dot{x}(t) \\
\dot{\lambda}(t)
\end{array}\right]=\left[\begin{array}{cc}
A & -B R^{-1} B^{T} \\
-C^{T} Q C & -A^{T}
\end{array}\right]\left[\begin{array}{l}
x(t) \\
\lambda(t)
\end{array}\right]+\left[\begin{array}{l}
G(t) \\
C^{T} Q r(t)
\end{array}\right]} \\
& x\left(t_{0}\right)=x_{0} \\
& \lambda\left(t_{f}\right)=\Pi_{f} x\left(t_{f}\right) .
\end{aligned}
$$

Under the assumption of periodic, steady-state behavior, we consider the cost functional

$$
J(u)=\int_{t_{0}}^{\tau} \frac{1}{2}\left[(C x(t)-r(t))^{T} Q(C x(t)-r(t))+u^{T}(t) R u(t)\right] d t
$$

where $\tau$ is the largest commensurate frequency.

Since the input operator $B$ is linear, a fundamental solution matrix is determined by solving the algebraic Ricatti equation

$$
A^{T} \Pi+\Pi A-\Pi B R^{-1} B^{T} \Pi+C^{T} Q C=0
$$

The optimal control input is then defined by

$$
u^{*}(t)=-R^{-1} B^{T}[\Pi x(t)-\nu(t)]
$$


where the variable $\nu(t) \in \mathbb{R}^{2 N}$ is the solution to the auxillary differential equation

$$
\begin{aligned}
& \dot{\nu}(t)=-\left[A-B R^{-1} B^{T} \Pi\right]^{T} \nu(t)-C^{T} \operatorname{Qr}(t)+\Pi G(t) \\
& \nu\left(t_{f}\right)=C^{T} \operatorname{Pr}\left(t_{f}\right) .
\end{aligned}
$$

The use of the final time condition results from approximation of the periodic boundary conditions to facilitate implementation. Details regarding this approach are given in $[33,34]$. The control trajectory determined by Eq. (35) is used in the following numerical examples to illustrate the need for nonlinear control when the input field reaches a magnitude that induces hysteresis.

\subsubsection{Numerical Results: Linear Model}

We demonstrate tracking control using the linear control theory to illustrate the performance in tracking a reference signal when the applied field level is either small or moderate to large. In the simulations, we apply a large input field to the rod model to simulate magnetization of the Terfenol-D rod prior to control simulation. The set of parameters given in Table 1 were used in the simulations. The values for the penalities on the observable state and input were $Q=1 \times 10^{12}$ and $R=1 \times 10^{-10}$. The step-size used in all simulations was $\Delta t=0.01 \mathrm{~s}$.

In all simulations, the disturbance forces $F_{d}$ were set to zero. It was determined that loads in excess of the coercive stress were necessary to induce noticeable disturbance vibrations relative to the tracking displacement. This eliminated the need to determine the optimal input for disturbance load compensation for the system under consideration.

Linear tracking control is demonstrated using a reference signal that starts at zero, ramps up, oscillates at a specified frequency and then ramps down to zero in accordance with the machining procedure described in the Introduction. The control is turned on immediately at $t_{o}=0$. In Fig. 4 , the reference signal is limited to small displacements so that the constitutive behavior is linear. In this case, excellent tracking is achieved as expected.

When the reference signal is increased to a level that induces hysteretic magnetic field-magnetization behavior, degradation in control authority occurs. Fig. 5(a) illustrates large error between the reference signal and the controlled displacement which clearly does not achieve the 1-2 $\mu \mathrm{m}$ tolerances dictated by the machining application discussed in the Introduction. The corresponding hysteretic $H-M$ behavior is illustrated in Fig. 5(b). 


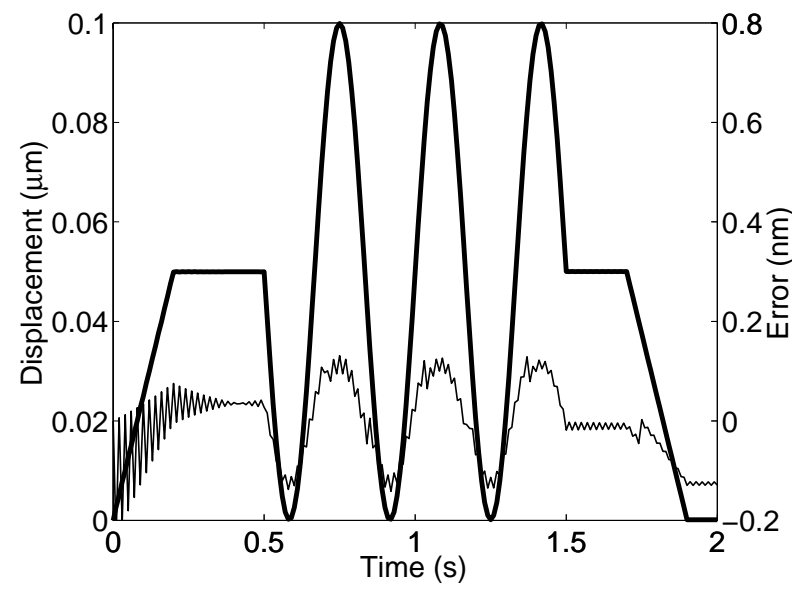

(a)

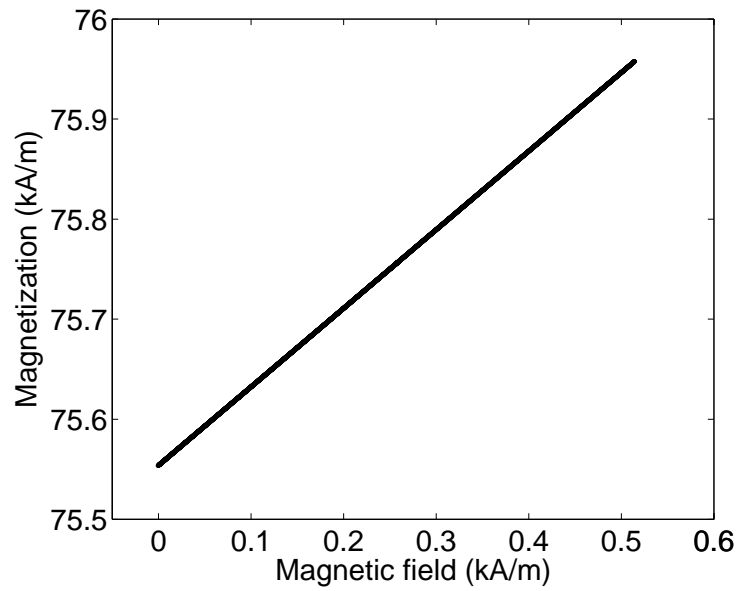

(b)

Figure 4: (a) Tracking control response when the magnetostrictive material behavior is limited to the linear regime. (b) The $H-M$ behavior was computed using Eq. (6).

\subsection{Nonlinear Optimal Tracking}

Accruate tracking in the presence of input nonlinearities and hysteresis is addressed in this section by implementing the constitutive behavior directly into the optimality system given by Eqs. (18) and (25). The methodology follows a previous approach for active vibration attenuation of beams and plates [13, 14]. Key

Table 1: Parameters used in the tracking control problem.

\begin{tabular}{l|l}
\hline Magnetostrictive and Damped Oscillator Parameters \\
\hline$k_{L}=1.5 \times 10^{7} \mathrm{~N} / \mathrm{m}$ & $Y^{M}=3.0 \times 10^{10} \mathrm{~N} / \mathrm{m}^{2}$ \\
$c_{L}=1.5 \times 10^{3} \mathrm{Ns} / \mathrm{m}$ & $A=5.0 \times 10^{-4} \mathrm{~m}^{2}$ \\
$m_{L}=0.47 \mathrm{~kg}$ & $L=0.1 \mathrm{~m}$ \\
$a_{1}=1.5 \times 10^{-4} \mathrm{~N} / \mathrm{A}^{2}$ & $c_{D}=3.7 \times 10^{6} \mathrm{Ns} / \mathrm{m}$ \\
$c_{1}=6.1 \times 10^{-5} \mathrm{~m} / \mathrm{A}$ & $b=1.5 \times 10^{4} \mathrm{~A} / \mathrm{m}$ \\
$\bar{H}_{c}=3.3 \times 10^{3} \mathrm{~A} / \mathrm{m}$ & $c=0.65$ \\
$M_{R}=3.7 \times 10^{4} \mathrm{~A} / \mathrm{m}$ & $\chi_{M}=15$ \\
$M_{0}=2.75 \times 10^{5} \mathrm{~A} / \mathrm{m}$ & \\
\hline
\end{tabular}




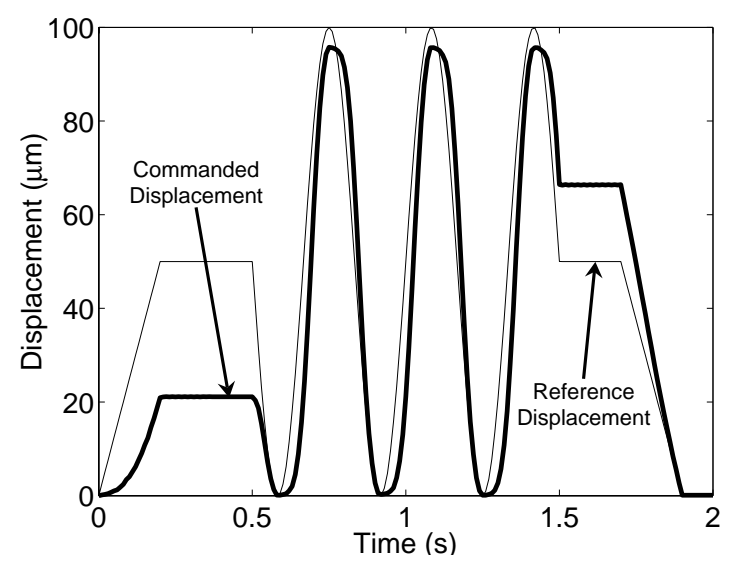

(a)

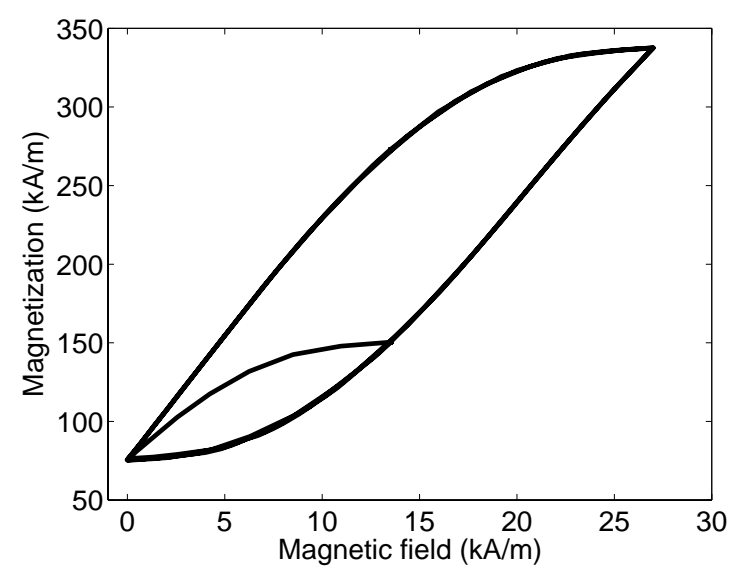

(b)

Figure 5: Linear tracking control response when magnetostrictive nonlinearities and hysteresis are present. (a) The controlled response is given by (-) and the reference signal is given by (-). (b) Corresponding constitutive behavior of the magnetostrictive transducer from the optimal linear control input.

equations are given to highlight modifications needed to track a reference signal.

In the nonlinear model, the force determined from Eq. (28) is included in the input operator $[B(u)](t)$ where the force generated by the magnetic field has been linearized about the biased field $M_{0}$. Whereas this approximation neglects the quadratic $M-\sigma$ relation in Eq. (11), it includes nonlinear and hysteretic $H-M$ relations. Further discussion regarding this approximation are given in sections 4.2.1 and 4.3.

The resulting optimality system is

$$
\begin{aligned}
& {\left[\begin{array}{c}
\dot{x}(t) \\
\dot{\lambda}(t)
\end{array}\right]=\left[\begin{array}{l}
A x(t)+[B(u)](t)+G(t) \\
-A^{T} \lambda(t)-C^{T} Q C x(t)+C^{T} Q r(t)
\end{array}\right]} \\
& x\left(t_{0}\right)=x_{0} \\
& \lambda\left(t_{f}\right)=C^{T} P\left(C x\left(t_{f}\right)-r\left(t_{f}\right)\right) .
\end{aligned}
$$

An efficient Ricatti formulation is not possible in this case due to the nonlinear nature of the input operator. The two-point boundary value problem is addressed by approximating Eq. (37) or the equivalent first-order system 


$$
\begin{aligned}
& \dot{z}(t)=F(t, z) \\
& E_{0} z\left(t_{0}\right)=\left[x_{0}, 0\right]^{T} \\
& E_{f} z\left(t_{f}\right)=[0,0]^{T}
\end{aligned}
$$

where $z=[x(t), \lambda(t)]^{T}$.

The nonlinear optimality system is now given by

$$
\begin{aligned}
& F(t, z)=\left[\begin{array}{l}
A x(t)+[B(u)](t)+G(t) \\
-A^{T} \lambda(t)-C^{T} Q C x(t)+C^{T} Q r(t)
\end{array}\right] \\
& E_{0}=\left[\begin{array}{ll}
\mathbb{I} & 0 \\
0 & 0
\end{array}\right], \quad E_{f}=\left[\begin{array}{cc}
0 & 0 \\
-C^{T} P C & \mathbb{I}
\end{array}\right] .
\end{aligned}
$$

Here II denotes an identity matrix with dimension corresponding to the number of basis functions employed in the spatial approximation of the state variables.

Various methods are available to approximate solutions to the system given by Eq. (38) such as finite differences and nonlinear multiple shooting [35]. The finite difference approach is used here where we consider a discretization of the time interval $\left[t_{0}, t_{f}\right]$ with a uniform mesh having stepsize $\Delta t$ and points $t_{0}, t_{1}, \cdots, t_{N}=t_{f}$. The approximate values of $z$ at these times are denoted by $z_{0}, \cdots, z_{N}$. A central difference approximation of the temporal derivative then yields the system

$$
\begin{aligned}
& \frac{1}{\Delta t}\left[z_{j+1}-z_{j}\right]=\frac{1}{2}\left[F\left(t_{j}, z_{j}\right)+F\left(t_{j+1}, z_{j+1}\right)\right] \\
& E_{0} z_{0}=\left[y_{0}, 0\right]^{T} \\
& E_{f} z_{N}=[0,0]
\end{aligned}
$$

for $j=0, \cdots, N-1$.

Equation (40) can be expressed as the problem of finding $z_{h}=\left[z_{0}, \cdots, z_{N}\right]$ which solves

$$
\mathcal{F}\left(z_{h}\right)=0
$$

Equation (41) includes the optimality system at each time step and the boundary conditions. Details are given in [13].

A quasi-Newton iteration of the form 


$$
z_{h}^{k+1}=z_{h}^{k}+\xi_{h}^{k}
$$

where $\xi_{h}^{k}$ solves

$$
\mathcal{F}^{\prime}\left(z_{h}^{k}\right) \xi_{h}^{k}=-\mathcal{F}\left(z_{h}^{k}\right)
$$

is then used to approximate the solution to the nonlinear system given by Eq. (41). The Jacobian $\mathcal{F}^{\prime}\left(z_{h}^{k}\right)$ has the form

$$
\mathcal{F}^{\prime}\left(z_{h}\right)=\left[\begin{array}{cccccc}
S_{0} & R_{0} & & & & \\
& S_{1} & R_{1} & & & \\
& & \ddots & \ddots & & \\
& & & & S_{N-1} & R_{N-1} \\
& & & & & E_{f}
\end{array}\right]
$$

where

$$
S_{i}=-\frac{1}{\Delta t}\left[\begin{array}{cc}
I & 0 \\
0 & I
\end{array}\right]-\frac{1}{2}\left[\begin{array}{cc}
A & \frac{\partial}{\partial \lambda} B\left[u_{i}^{*}\right] \\
-C^{T} Q C & -A^{T}
\end{array}\right]
$$

for $S_{i}$. The representation for $R_{i}$ is similar.

Inversion of the Jacobian is required to obtain updates to the states through the iteration procedure. Direct inversion is not feasible when a large number of basis functions are required to discretize the structural model. In the one-dimensional rod model, this is typically not an issue, although to reduce potential numerical errors in inverting the Jacobian an analytic LU decomposition is employed. The form of the equation is identical to that used in previous investigations $[13,14]$ and is given here in the Appendix for convenience. This gives rise to the following representation of the Jacobian

$$
\mathcal{F}^{\prime}\left(z_{h}^{k}\right)=L U
$$

where $L$ and $U$ respectively denote lower and upper triangular matrices.

The solution of the system defined by Eq. (43) is then obtained through direct solution of the lower triangular system $L \zeta_{h}^{k}=-\mathcal{F}\left(z_{h}^{k}\right)$ followed by direct solution of the upper triangular system $U \xi_{h}^{k}=\zeta_{h}^{k}$. This leads to updates to the state and adjoint soutions $z_{h}^{k+1}$. 


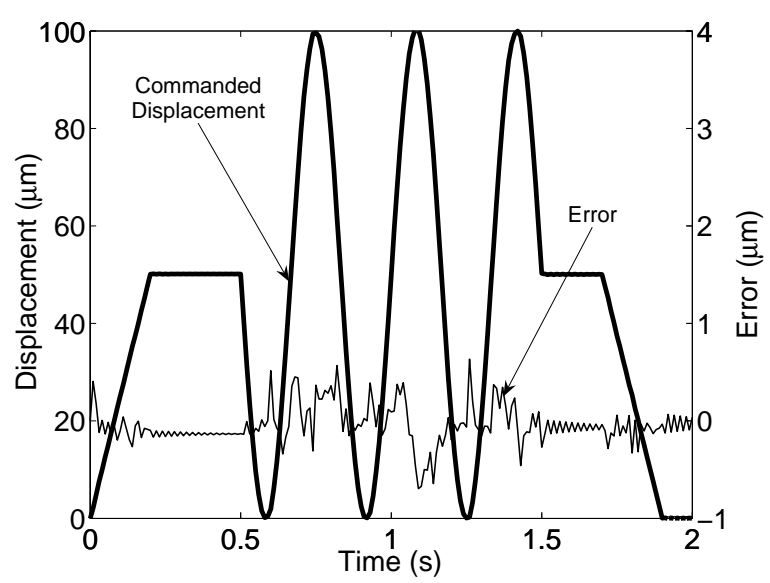

(a)

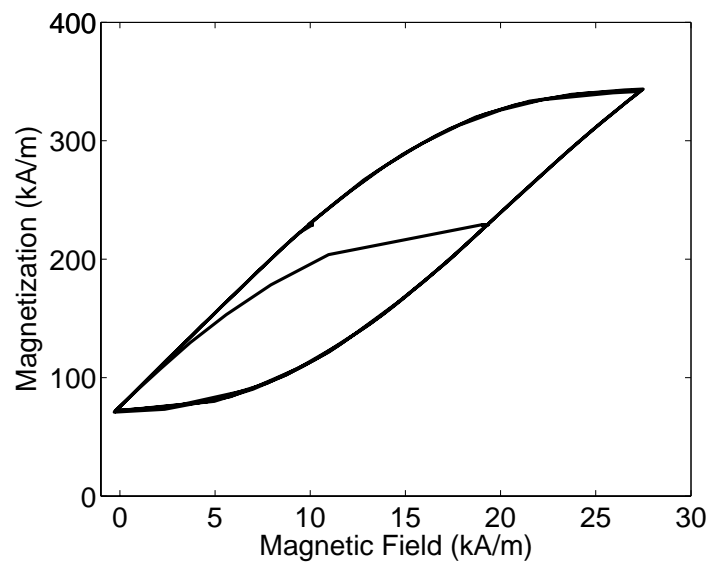

(b)

Figure 6: Tracking control response using the nonlinear control law. (a) The controlled response (in $\mu \mathrm{m})(\mathbf{m})$ and the error between the reference signal and controlled response (in $\mu \mathrm{m}$ ) (-). (b) Corresponding $H-M$ constitutive behavior.

\subsubsection{Nonlinear Simulation Results}

The nonlinear optimal tracking design provides enhanced control authority when nonlinearities and hysteresis are present. This is demonstrated by prescribing the same reference signal that induced hysteresis in Fig. 5(b) and poor tracking in Fig. 5(a). The transducer nonlinearities are effectively accommodated using the nonlinear optimal control method as shown in Fig. 6. In this simulation, the optimal control input was computed assuming that the actuation force was proportional to the magnetization as previously given by Eq. (28). In Fig. 6, the magnetostrictive actuator displacement was determined from the control input using the quadratic magnetization coupling as given by Eq. (11). This illustrates that the error from unmodeled quadratic coupling is minimal for a $100 \mu m$ reference trajectory. The same penalties on the observable state and control input from the linear simulations have been used with the additional penalty on the final state given by $P=C \Pi C^{T}$.

Remark 1: In the present model, nonlinearities were included in the input operator contained within the optimality system, Eq. (37). It should be noted that this method neglects nonlinearities contained within the components of the Jacobian (Eq. (45)) and the optimal input equation (Eq. (26)). The analytic form of the term $\frac{\partial}{\partial \lambda} B\left[u_{i}^{*}\right]$ in Eq. (45) requires explicit knowledge fo the Lagrange multipliers and the input which is unknown in the nonlinear case. This component is assumed to be constant which results in a suboptimal controller with $S_{i}=S$ and $R_{i}=R$ for each time step. It should also be noted that optimal input contains nonlinearities in 
the term defined by $\frac{\partial B^{T}(u)}{\partial u}$. The nonlinear term requires computing the tangential magnetic susceptibility, $\frac{\partial M}{\partial u}$. Since the input only induces minor hysteresis in the present analysis, changes in the magnetic susceptibility are expected to be small and are neglected in the nonlinear control simulations. The tangential magnetic susceptiblity is assumed to be equivalent to the linear coefficient $\chi_{m}=\frac{\partial M}{\partial u}$.

Remark 2: The control design presented here has focused on open loop nonlinear control that is computed off-line. It is well known that uncertainties in operating conditions or unmodeled dynamics can have deleterious effects on control performance. This is addressed in the following section using perturbation feedback. The approach is similar to a hybrid nonlinear optimal control design for attenuating plate vibration [14]. While there are similarities to the tracking problem, fundamental differences in the auxillary equation (Eq. (36)) are addressed to accurately track the prescribed reference signal when operating uncertainties are present.

\subsection{Perturbation Control and Estimation}

In the previous section, nonlinear open loop control was shown to provide excellent tracking behavior by compensating for nonlinear and hysteretic field-coupled magnetostrictive material behavior. However, any unmodeled dynamics not accounted for in the constitutive model or damped oscillator are a source of operating uncertainty which can be detrimental in obtaining high accuracy tracking. Moreover, external disturbances or slight delays in the control electronics can degrade control authority. These issues are addressed by implementing perturbation feedback around the optimal open loop trajectory computed in the preceding section.

As previously noted, the system under consideration contains unmeasurable states defined by the output equation (Eq. (22)) since it has been assumed that only the displacement at the end of the cutting tool is known. State feedback requires availability of all the states which necessitates the estimate of the unmeasurable states. This is accomplished by employing a Kalman filter. The estimated perturbations are then used to determine first-order variations in the optimal control input. The development of the perturbation controller and estimator forms the well-known linear quadratic Gaussian (LQG) control problem where incomplete state information is used to determine the LQ regulator described in Section 4.1 except the control is determined from feedback from the state estimates using the Kalman filter. Key equations used in developing the perturbation controller and Kalman filter are given here. Additional details can be found in [14, 36, 37].

The perturbed system consists of first-order variations in the constraint Eq. (18), output Eq. (22) and initial conditions, 


$$
\begin{aligned}
& \delta \dot{x}(t)=A \delta x(t)+B \delta u(t)+\delta G(t) \\
& \delta y(t)=C \delta x(t)+w(t) \\
& \delta x\left(t_{0}\right)=\delta \bar{x}_{0},
\end{aligned}
$$

where $\delta u, \delta x$ and $\delta y$ are first-order variations about $u^{*}, x^{*}$ and $y^{*}$. The nonlinear input operator $B(u)$ has been linearized around the optimal input $u^{*}(t)$. The perturbation in plant disturbances is denoted by $\delta G(t)$. In comparison to the output in Eq. (22), measurement noise $w(t)$ has been included in the first-order variation of the output to illustrate robustness in the resulting design. Both the variation in system noise $\delta G(t)$ and the measurement noise $w(t)$ are assumed to be random processes with zero mean. The covariance for the system and measurement noise is defined by $E\left[\delta G(t) \delta G^{T}(\tau)\right]=V \delta(t-\tau)$ and $E\left[w(t) w^{T}(\tau)\right]=W \delta(t-\tau)$ with the joint property $E\left[\delta G(t) w^{T}(\tau)\right]=0$.

The cost functional given by Eq. (23) is used to determine the optimal perturbation feedback. Since the optimal control pair $\left(u^{*}(t), x^{*}(t)\right)$ minimizes $\delta \bar{J}$, the second order terms are expanded to determine $\left(\delta u^{*}(t), \delta x^{*}(t)\right)$. The second order variation in $\bar{J}$ is given by

$$
\delta^{2} \bar{J}=\frac{1}{2} \int_{t_{0}}^{t_{f}}\left[\begin{array}{ll}
\delta x^{T} & \delta u^{T}
\end{array}\right]\left[\begin{array}{ll}
\bar{Q} & 0 \\
0 & R
\end{array}\right]\left[\begin{array}{l}
\delta x \\
\delta u
\end{array}\right] d t
$$

where penalties on the perturbed states and input are given by $\bar{Q}$ and $R$, respectively. Here, $\bar{Q} \in \mathbb{R}^{2 N \times 2 N}$ is a matrix that weights both the displacement and velocity at the end of the cutting tool. This minimizes the potential for large variations in the cutting tool velocity. A similar approach is given in [30]. The form of $\delta x^{T} \bar{Q} \delta x$ is given by

$$
\delta x^{T} \bar{Q} \delta x=q_{1} \delta x_{N}^{2}+q_{2} \delta x_{2 N}^{2}
$$

where $\delta x_{N}$ is the variation in displacement at the tip of the cutting tool and $\delta x_{2 N}$ is the variation in velocity at the tip of the cutting tool. The parameters $q_{1}$ and $q_{2}$ are the respective penalties.

Due to the form of the performance index given by Eq. (48), previously discussed LQR theory can be directly employed to obtain $\delta u^{*}(t)$ and $\delta x^{*}(t)$. The overall control for the system is then taken to be $u^{*}(t)+\delta u^{*}(t)$ with the optimal state given by $x^{*}(t)+\delta x^{*}(t)$. The perturbed feedback signal is given by

$$
\delta u^{*}(t)=-R^{-1} B^{T}[\Pi \delta \hat{x}(t)-\delta \nu(t)]
$$


where $\Pi$ is the solution of the algebraic Ricatti equation for the perturbed system previously given by Eq. (34). The variable $\delta \hat{x}(t)$ is the estimate of the first-order variations in the states given by Eq. (47) which will be subsequently determined from the Kalman filter.

The variable $\delta \nu(t)$ given in Eq. (50) is a feedforward term used to compensate for phase delays or periodic external disturbances. It is governed by the auxilliary equation

$$
\begin{aligned}
& \delta \dot{\nu}(t)=-\left[A-B R^{-1} B^{T} \Pi\right]^{T} \delta \nu(t)-C^{T} Q \delta r(t)+\Pi \delta G(t) \\
& \delta \nu\left(t_{f}\right)=C^{T} P \delta r\left(t_{f}\right) .
\end{aligned}
$$

where the final time conditions are zero in the present machining example.

To solve Eq. (51), the perturbation in the disturbance $\delta G(t)$ and reference signal $\delta r(t)$ must be known or estimated. The perturbation in the reference signal is defined by $\delta r(t)=y(t)-r(t+\delta t)$ where the time shift is denoted by $\delta t$. As mentioned previously, disturbance loads have a negligible effect on tracking performance, therefore perturbations in the disturbance loads $\delta G(t)$ are not considered in the simulations.

The perturbation feedback control given by Eq. (50) requires estimation of the states in Eq. (47) which are given by the relation

$$
\begin{aligned}
& \delta \dot{\hat{x}}(t)=A \delta \hat{x}(t)+B \delta u(t)+L(t)[\delta y(t)-C \delta \hat{x}(t)] \\
& \delta \hat{x}\left(t_{0}\right)=\delta \hat{x}_{0}
\end{aligned}
$$

where $\delta \hat{x}_{0}$ is the estimated initial conditions and $L(t)$ is the Kalman gain.

The Kalman gain is determined from the dynamics of the error between the first-order variation in the actual states and the estimated states. Key equations used in determining $L(t)$ are given here. Additional details can be found in $[36,37]$.

The Kalman gain is determined from the error covariance $\Upsilon(t)=E\left[e(t) e^{T}(\tau)\right]$ where the error is determined from the dynamic relation

$$
\dot{e}(t)=\delta \dot{x}(t)-\delta \dot{\hat{x}}(t)
$$

By substituting Eqs. (47) and (52) into Eq. (53) the error is

$$
e(t)=\Phi\left(t, t_{0}\right) e\left(t_{0}\right)+\int_{t_{0}}^{t} \Phi(t, \tau)[\delta G(\tau)-L(\tau) w(\tau)] d \tau
$$

where $\Phi\left(t, t_{0}\right)$ is the state transition matrix. Through manipulation of $\Upsilon(t)$ outlined in [36, 37] the rate of change of the error covariance leads to the differential matrix Ricatti equation 


$$
\dot{\Upsilon}(t)=A \Upsilon(t)+\Upsilon(t) A^{T}-\Upsilon(t) C^{T} W^{-1} C \Upsilon(t)+V
$$

with the initial conditions $\Upsilon\left(t_{0}\right)=\Upsilon_{0}$. The Kalman gain is found by maximizing the negative rate of change of $\Upsilon(t)$ which leads to

$$
L(t)=\Upsilon(t) C^{T} W^{-1}
$$

In the simulations presented here, a steady-state Kalman gain $L(t)=L$ is determined by solving the algebraic form of Eq. (55) where $\dot{\Upsilon}(t)=0$. This leads to an approximately optimal system that is easier to implement numerically.

In summary, the matrix system governing the dynamics of the perturbed optimality system

$$
\begin{aligned}
& {\left[\begin{array}{c}
\delta \dot{x}(t) \\
\delta \dot{\hat{x}}(t)
\end{array}\right]=\left[\begin{array}{cc}
A & -B R^{-1} B^{T} \Pi \\
L C & A-B R^{-1} B^{T} \Pi-L C
\end{array}\right]\left[\begin{array}{c}
\delta x(t) \\
\delta \hat{x}(t)
\end{array}\right]+\left[\begin{array}{c}
B R^{-1} B^{T} \\
B R^{-1} B^{T}
\end{array}\right] \delta \nu(t)+\left[\begin{array}{c}
\delta G(t) \\
L w(t)
\end{array}\right]} \\
& \delta x\left(t_{0}\right)=\delta x_{0} \\
& \delta \hat{x}\left(t_{0}\right)=\delta \hat{x}_{0} .
\end{aligned}
$$

is solved to obtain the system response when perturbation feedback of the estimated states is implemented.

\subsubsection{Numerical Results}

The effectiveness of the perturbation controller and estimator is illustrated by applying an initial phase delay of $\delta t=0.03 \mathrm{~s}$ to the Terfenol-D transducer to model the effects of delays in the electronics. The tracking performance is compared to the cases when measurement noise is either negligible or significant. In these simulations, the system response was assessed when the measurement noise was corrupted by a signal with a $100 \mu \mathrm{m}$ amplitude and compared to a baseline case when $w(t)=0$. As previously noted, whereas disturbance loads are included in the governing equations, these forces were neglected in the simulations since large stresses in excess of the coercive stress were necessary to induce undesirable vibration in the rod. A similar approach is taken here where perturbations in the disturbance forces are neglected. The quantities used for the penalties in the second variation of the cost functional were $q_{1}=1 \times 10^{16}, q_{2}=1 \times 10^{11}$ and $R=1 \times 10^{-12}$. The Kalman filter gain was computed using a value of $V=1 \times 10^{8}$ as a design parameter for the covariance of $\delta G(t)$ and $W=1 \times 10^{-7}$ for the covariance of $w(t)$. 
The system performance with and without perturbation feedback is illustrated in Figs. 7 and 8, respectively. As expected, the open loop tracking control in Fig. 7 results in an offset in displacement shifted by $0.03 \mathrm{~s}$ relative to the desired reference trajectory leading to large errors in tracking the reference signal. Significant improvements are made when perturbation feedback control is employed. Figure 8 compares tracking performance when the measurement noise is zero (Fig. 8(a)) to performance when the noise is nonzero (Fig. 8(b)). Whereas the error is initially large from the phase shift, it subsequently reduces to values comparable to the open loop case when delays in the electronics are absence (Fig. 6) except at points where abrupt transitions in displacement occur. When measurement noise is present, the Kalman filter limits the error to approximately the amplitude of $w(t)$ as illustrated in Fig. 8(b).

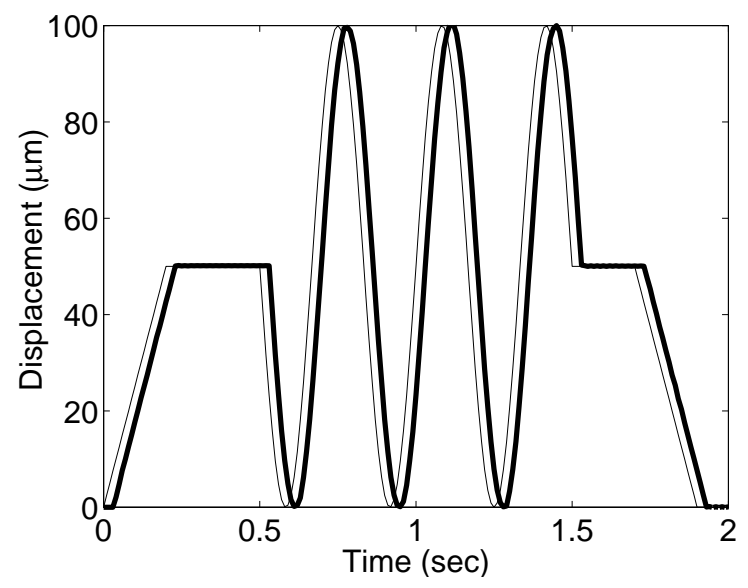

Figure 7: The effect of an initial time delay in the open loop control signal is illustrated where ( $\longrightarrow$ ) is the controlled response and (-) is the reference signal. 


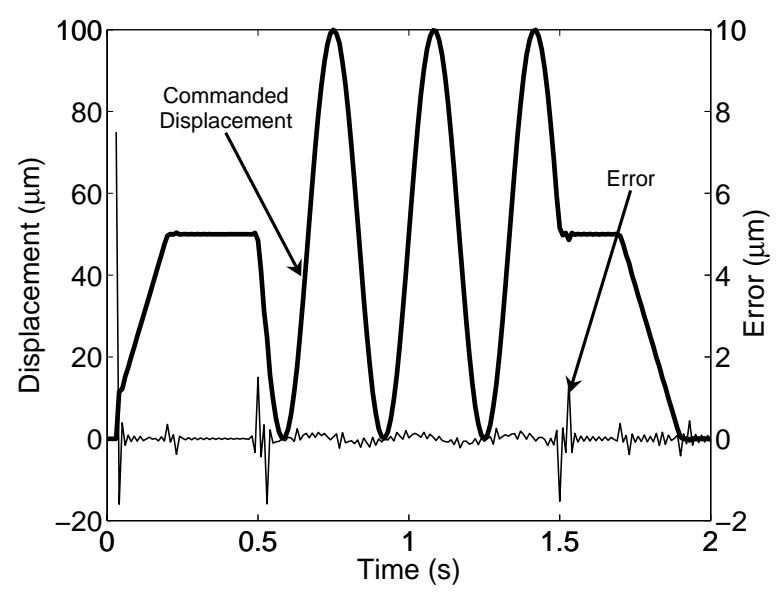

(a)

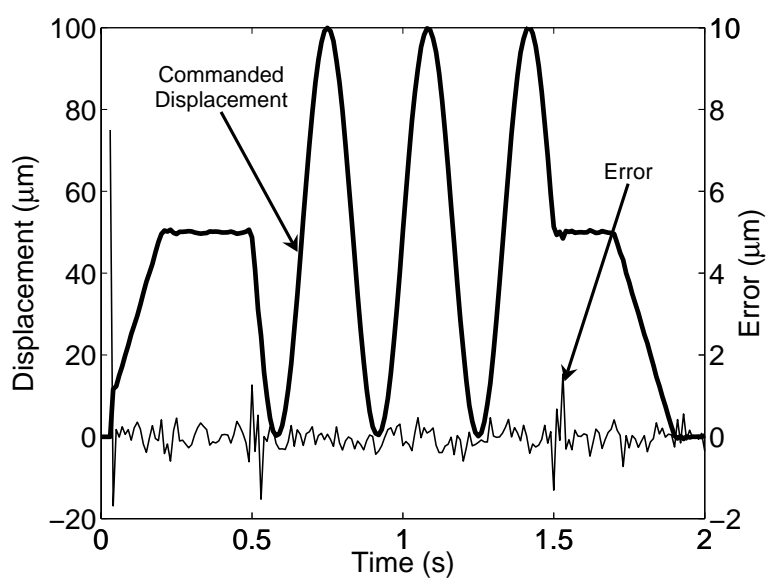

(b)

Figure 8: Perturbation feedback using the estimated states from the Kalman filter: (a) measurement noise is zero, and (b) measurement noise is a random signal with an amplitude of $1 \mu \mathrm{m}$.

\section{Concluding Remarks}

A model-based nonlinear optimal control design was developed to accurately track a reference signal when nonlinear, hysteretic magnetostrictive material behavior is present. Whereas linear control was effective for tracking the desired reference signal for small displacements, significant errors occur at moderate to high field inputs if the constitutive behavior is not properly accommodated. This was addressed by incorporating the homogenized energy model into the control design to compensate for nonlinear, hysteretic field-coupled magnetostrictive constitutive behavior. The open loop nonlinear control method provided excellent tracking performance at field levels where nonlinearities and hysteresis are present. Although this enhanced performance, open loop control is not practical in applications due to uncertainties in operating conditions. This issue was addressed by complementing the open loop nonlinear control with linear perturbation feedback using LQG control. This hybrid control technique included a Kalman filter in the perturbed optimality system to feedback estimations of variations in states around the optimal open loop trajectory. This approach provides a potential method for real-time control when certain states are unobservable by computing and storing the open loop nonlinear control and applying real-time linear feedback of the perturbed states.

The nonlinear tracking control design has focused on compensating for rate-independent, nonlinear and hysteretic magnetostrictive material behavior, although the control design is general for a broad class of ferroic materials due to the unified properties on the homogenized energy model. The constitutive model has been 
successful in modeling the behavior of ferroelectric materials and shape memory alloys [25]. Moreover, the homogenized energy framework is applicable to rate-dependent hysteresis in materials where thermal relaxation mechanisms are present or the drive frequency affects the level of hysteretic losses. Implementing this behavior into nonlinear control designs is under current investigation.

\section{Acknowledgments}

The authors gratefully acknowledge support from the Air Force Office of Scientific Research through grant AFOSR-FA9550-04-1-0203.

\section{Appendix}

\section{A.1 Matrix and Vector Relations}

The linear interpolation functions used to formulate the matrices and vectors given in Section 3.1 are given here. By substituting the approximate displacements in Eq. (16) into the variational form of the dynamic equation, Eq. (13) has the form

$$
\begin{aligned}
\sum_{j=1}^{N} \ddot{w}_{j}(t) \int_{0}^{L} \rho A \phi_{i} \phi_{j} d x+ & \sum_{j=1}^{N} \dot{w}_{j}(t) \int_{0}^{L} c_{D} A \phi_{i}^{\prime} \phi_{j}^{\prime} d x+\sum_{j=1}^{N} w_{j}(t) \int_{0}^{L} Y^{M} A \phi_{i}^{\prime} \phi_{j}^{\prime} d x=F_{m a g}(H) \int_{0}^{L} \phi_{i}^{\prime} d x \\
& +\int_{0}^{L} F_{d} \phi_{i} d x-\left(k_{L} w_{N}(t) \phi_{N}(L)+c_{L} \dot{w}_{N}(t) \phi_{N}(L)+m_{L} \ddot{w}_{N} \phi_{N}(L)\right) \phi_{N}(L) .
\end{aligned}
$$

By comparing the above relation to Eq. (17), the global mass, stiffness, and damping matrices contain the components

$$
\begin{aligned}
& {[\mathbb{M}]_{i j}= \begin{cases}\int_{0}^{L} \rho A \phi_{i} \phi_{j} d x, & i \neq N \text { or } j \neq N \\
\int_{0}^{L} \rho A \phi_{i} \phi_{j} d x+m_{L}, & i=N \text { or } j=N\end{cases} } \\
& {[\mathbb{C}]_{i j}= \begin{cases}\int_{0}^{L} c_{D} A \phi_{i}^{\prime} \phi_{j}^{\prime} d x, & i \neq N \text { or } j \neq N \\
\int_{0}^{L} c_{D} A \phi_{i}^{\prime} \phi_{j}^{\prime} d x+c_{L}, & i=N \text { or } j=N\end{cases} }
\end{aligned}
$$




$$
[\mathbb{K}]_{i j}= \begin{cases}\int_{0}^{L} Y^{M} A \phi_{i}^{\prime} \phi_{j}^{\prime} d x, & i \neq N \text { or } j \neq N \\ \int_{0}^{L} Y^{M} A \phi_{i}^{\prime} \phi_{j}^{\prime} d x+k_{L}, & i=N \text { or } j=N\end{cases}
$$

whereas the force vectors have the components

$$
\left[\vec{f}_{d}\right]_{i}=\int_{0}^{L} F_{d} \phi_{i} d x \quad, \quad[\vec{b}]_{i}=\int_{0}^{L} \phi_{i}^{\prime} d x
$$

\section{A.2 LU Decomposition}

The Jacobian given by Eq. (44) is written in terms of a LU decomposition to avoid matrix inversion when considering large systems. The analytic form of the decomposition used in the simulations is

$$
\begin{aligned}
& L=\left[\begin{array}{ccccc}
S_{0} & & & & \\
& S_{1} & & & \\
& & & & \\
& & \ddots & & \\
& & & S_{N-1} & 0 \\
E_{0} & -E_{0}\left(S_{0}^{-1} R_{0}\right) & \cdots & E_{0} \prod_{i=0}^{N-2}(-1)^{i}\left(S_{i}^{-1} R_{i}\right) & E_{f}+E_{0} \prod_{i=0}^{N-1}(-1)^{i}\left(S_{i}^{-1} R_{i}\right)
\end{array}\right] \\
& U=\left[\begin{array}{ccccc}
I & S_{0}^{-1} R_{0} & & & \\
& I & S_{1}^{-1} R_{1} & & \\
& & \ddots & \ddots & \\
& & & I & S_{N-1}^{-1} R_{N-1} \\
& & & & I
\end{array}\right] .
\end{aligned}
$$

This form of the matrix system reduces the computation to solving $2 N \times 2 N$ systems for each time step separately. This provides the ability to solve matrix systems containing a large number of degrees of freedom while maintaining a small temporal step size. 


\section{References}

[1] Xu, B., and L. E. Cross, J. J. B., 2000. "Ferroelectric and antiferroelectric films for microelectromechanical systems applications". Thin Solid Films, 377-378, pp. 712-718.

[2] Straub, F., Kennedy, D., Domzalski, D., Hassan, A., Ngo, H., Anand, V., and Birchette, T., 2004. "Smart material-actuated rotor technology—SMART". J. Intell. Mater. Syst. Struct., 15, pp. 249-260.

[3] Giessibl, F., 2003. "Advances in atomic force microscopy". Rev. Mod. Phys., 75, pp. 949-983.

[4] Schitter, G., Menold, P., Knapp, H., Allgower, F., and Stemmer, A., 2003. "High performance feedback for fast scanning atomic force microscopes". Rev. Sci. Instrum., 72(8), pp. 3320-3327.

[5] Dapino, M., 2004. "On magnetostrictive materials and their use in adaptive structures". Struct. Eng. Mech., 17(3-4), pp. 303-329.

[6] Dapino, M., 2002. "Magnetostrictive materials". In Encyclopedia of Smart Materials, M. Schwartz, ed. John Wiley and Sons, New York, pp. 600-620.

[7] Nealis, J., and Smith, R. "Model-based robust control design for magnetostrictive transducers operating in hysteretic and nonlinear regimes". submitted to IEEE Trans. Control Syst. Technol.

[8] Dapino, M., Calkins, F., and Flatau, A., 1999. "Magnetostrictive devices". In Wiley Encyclopedia of Electrical and Electronics Engineering, J. Webster, ed., Vol. 12. John Wiley and Sons, New York, pp. 278305.

[9] Kudva, J., 2004. "Overview of the DARPA smart wing project". J. Intell. Mater. Syst. Struct., 15(4), pp. 261-267.

[10] Kudva, J., Sanders, B., Pinkerton-Florance, J., and Garcia, E., 2002. "The DARPA/AFRL/NASA smart wing program-final overview". Proc. SPIE Int. Soc. Opt. Eng., 4698, pp. 37-43.

[11] Calkines, F., and Butler, G., 2004. "Subsonic jet noise reduction variable geometry chevron". Proc. 42nd AIAA Aerospace Sciences Meeting and Exhibit.

[12] Tao, G., and Kokotović, P., 1996. Adaptive control of systems with actuator and sensor nonlinearities. John Wiley and Sons, New Jersey.

[13] Smith, R., 1995. "A nonlinear optimal control method for magnetostrictive actuators". J. Intell. Mater. Syst. Struct., 9(6), pp. 468-486. 
[14] Oates, W., and Smith, R. "Nonlinear optimal control techniques for vibration attenuation using nonlinear magnetostricive actuators". submitted to J. Intell. Mater. Sys. Struct.

[15] Hiller, M., Bryant, M., and Umegaki, J., 1989. "Attenuation and transformation of vibration through active control of magnetostrictive Terfenol". J. Sound Vib., 134(3), pp. 507-519.

[16] Bryant, M., Fernández, B., Wang, N., Murty, V., Vadlamani, V., and West, T., 1992. "Active vibration control in structures using magnetostrictive terfenol with feedback and/or neural network controllers". Proceedings of the Conference on Recent Advances in Adaptive and Sensory Materials and their Applications, 5, pp. 431-436.

[17] Ge, P., and Jouaneh, M., 1996. "Tracking control of a piezoceramic actuator". IEEE T. Contr. Syst. T., 4(3), pp. 209-216.

[18] Grant, D., and Hayward, V., 1997. "Variable structure control of shape memory alloy actuators". IEEE Contr. Syst. Mag., 17(3), pp. 80-88.

[19] Smith, R., 2001. "Inverse compensation for hysteresis in magnetostrictive transducers". Math. Comput. Model., 33, pp. 285-298.

[20] Jiles, D., and Atherton, D., 1984. "Theory of the magnetomechanical effect". J. Phys. D. Appl. Phys., 17, pp. $1265-1281$.

[21] Jiles, D., 1992. "A self-consistent generalized model for the calculation of minor loop excursions in the theory of hysteresis". IEEE T. Magn., 28(5), pp. 2602-2604.

[22] Smith, R., 2005. Smart Material Systems: Model Development. SIAM, Philadelphia, PA.

[23] Smith, R., Seelecke, S., Ounaies, Z., and Smith, J., 2003. "A free energy model for hysteresis in ferroelectric materials". J. Intell. Mater. Syst. Struct., 14(11), pp. 719-739.

[24] Smith, R., Dapino, M., and Seelecke, S., 2003. "A free energy model for hysteresis in magnetostrictive transducers". J. Appl. Phys., 93(1), pp. 458-466.

[25] Smith, R., Seelecke, S., Dapino, M., and Ounaies, Z. "A unified framework for modeling hysteresis in ferroic materials". to appear in the J. Mech. Phys. Solids.

[26] Jiles, D., 1991. Introduction to Magnetism and Magnetic Materials. Chapman and Hall, New York.

[27] Hall, D., and Flatau, A., 1998. "Analog feedback control for magnetostrictive transducer linearization". J. Sound Vib., 211(3), pp. 481-494. 
[28] Flatau, A., and Kellog, R., 2004. "Blocked-force characteristics of Terfenol-D tranducers". J. Intell. Mater. Syst. Struct., 15, pp. 117-128.

[29] Dapino, M., Smith, R., and Flatau, A., 2000. "A structural strain model for magnetostrictive transducers". IEEE T. Magn., 36(3), pp. 545-556.

[30] Anderson, B., and Moore, J., 1990. Optimal Control: Linear quadratic Methods. Prentice Hall, Englewood Cliffs, NJ.

[31] Bryson, A., and Ho, Y.-C., 1969. Applied Optimal Control. Blasidell Publishing Company, Waltham, MA.

[32] Lewis, F., and Syrmos, V., 1995. Optimal Control. John Wiley and Sons, New York, NY.

[33] Bittanti, S., Locatelli, A., and Maffezzoni, C., 1972. "Periodic opimization under small perturbations". In Periodic Optimization, A. Marzollo, ed., Vol. II. Udine, Springer-Verlag, New York, pp. 183-231.

[34] DaPrato, G., 1987. "Synthesis of optimal control for an infinite dimensional periodic problem". SIAM J. Control Optim., 25(3), pp. 706-714.

[35] Ascher, U., Mattheij, R., and Russell, R., 1995. Numerical Solution of Boundary Value Problems for Ordinary Differential Equations. SIAM Classics in Applied Mathematics.

[36] Lewis, F., 1986. Optimal Estimation With an Introduction to Stochastic Control Theory. John Wiley and Sons, New York, NY.

[37] Bay, J., 1999. Fundamentals of Linear State Space Systems. WCB McGraw-Hill. 